\title{
Sustitución de cultivos ilícitos y cultura de la legalidad en Colombia* Illicit crop substitution and culture of legality in Colombia
}

\author{
Erika M. Rodríguez Pinzón** \\ Universidad Autónoma de Madrid y Universidad Internacional de la Rioja \\ erika.rodriguez@uam.es
}

Recibido / received: 31/12/2017

Aceptado / accepted: 28/01/2018

DOI: https://doi.org/10.20318/eunomia.2018.4159

\section{Resumen}

Este artículo analiza el problema de los cultivos ilícitos y concretamente de los programas de desarrollo alternativo destinados a solucionarla y su relación con la creación de la cultura de la legalidad.

En primer lugar analiza el desarrollo alternativo y recorre los diferentes programas que se han desarrollado en Colombia con el fin de conseguir la sustitución de cultivos ilícitos. Después se recogen algunos de los aspectos más relevantes de la relación entre cultivos ilícitos y fallas en la cultura de la legalidad.

La última parte del artículo recoge la evidencia empírica de diversos estudios y evaluaciones sobre el impacto de los diferentes programas en el fortalecimiento de la confianza, la participación y la cultura de la legalidad.

\section{Palabras clave}

Sustitución de cultivos, desarrollo alternativo, narcotráfico, Colombia, Cultura de la legalidad, Estado de Derecho

\begin{abstract}
This article analyzes the problematic of illicit crops in Colombia and, more specifically, the alternative development programs created to address it and how they are changing the culture of ilegality intrinsic to ilicit crops.

It analyzes the relation between a certain culture of illegality and the prevalence of narcotrafic; then it studies the presence of illicit crops in Colombia and describes the evolution of alternative development programs.

Finally, it collects and analyzes evidence from different empirical studies about the impact of alternative development programs in the enhancement of social confidence, strengthening of democracy and promotion of legality.
\end{abstract}

\footnotetext{
* Este artículo surge de una ponencia presentada en el IV Congreso de FLACSO ESPAÑA 2017 en la mesa: Cultura de la legalidad y cumplimiento de derechos en América Latina.

** Doctora por la Universidad Autónoma de Madrid (UAM). ORCID: 0000-0002-9004-8109
} 


\title{
Keywords
}

Illicit crop substitution, alternative development, narcotraffic, Colombia, Culture of legality, Rule of Law

\begin{abstract}
SUMARIO. 1. Introducción: Narcotráfico y debilidad del Estado Nación. 2. Narcotráfico y cultura de la legalidad. 3. El desarrollo alternativo como solución al problema de los cultivos ilícitos. 4. Presencia de los cultivos de Coca en Colombia. 4.1. La política dicotómica de la contención de los cultivos ilícitos: entre la seguridad y el desarrollo social. 4.2. Programas de Desarrollo Alternativo. 5. Cultura de la legalidad y substitución de cultivos ilícitos: Evidencia Empírica. 6. Conclusiones y perspectivas. 7. Bibliografía.
\end{abstract}

\section{Introducción: Narcotráfico y debilidad del Estado Nación}

Si bien, la lucha contra las drogas ilícitas se ha planteado de forma global, es necesario explicar por qué ha sido en Colombia donde ha tenido un impacto tan alto. La respuesta se encuentra, en primer lugar, en las bases de constitución del Estado, fragmentado, incapaz de controlar la periferia, y embarcado en una relación dialéctica con poderes facticos regionales que le proporcionan votos, pero a su vez, retan el poder del gobierno central cuando intenta controlarlos.

En segundo lugar, hay que señalar una causa y efecto relacionado pero menos visible, la brecha entre la sociedad y el Estado, en este caso representada por la desconfianza. Siguiendo a Pecaut (1994) la desconfianza respecto al Estado, no es una cosa nueva en Colombia, tiene sus raíces en una historia multisecular y de hecho, tiene su causa según en la droga dado que una gran parte de la sociedad, mucho mayor que aquella que está directamente implicada en el tráfico mismo, ha definido sus propias normas, sus redes de influencia, códigos de transacción, sin entenderse de las regulaciones estatales y jurídicas, como no sea para desviarlas en su propio beneficio. De esta forma, las fronteras entre lo legal y lo ilegal han desaparecido en muchos sectores.

El resultado, continúa Pecaut es que confrontada a esta situación el resto de la sociedad, se va quedando sin razones para confiar en un Estado ausente y autista. Así, se ha producido una suerte de emancipación de la sociedad, pero de una sociedad fragmentada ella misma, parcelada, rebelde por tanto a las regulaciones de cualquier gobierno, y en ese sentido, muy poco «civil».

La desconfianza y ruptura entre Estado y sociedad tiene una segunda esfera de implicaciones. El gobierno, por su parte, tiene que cumplir sus compromisos aceptados en materia de lucha antinarcóticos pero se ve incapaz de sobreponerse al problema, porque, es incapaz de reconocer que no es solo una actividad delictiva, sino que el narcotráfico está intrínsecamente ligado a la génesis misma del Estado y a las brechas entre este y la sociedad. El narcotráfico es consecuencia de una ruptura en la configuración misma del Estado y una causa de la profundización de este déficit. Pero no solo el narcotráfico juega este rol, también otras actividades que se generan en los márgenes del control estatal como la minería ilegal.

La economía de la droga ha propiciado un remezón de las estructuras sociales. Sus efectos no solo produjeron una bonanza de los sectores inmobiliario y financiero 
(esta último poco estudiado e infra dimensionado), sino que ha conllevado una auténtica "contrarreforma agraria". Por lo menos cuatro millones de hectáreas de las tierras más productivas del país han pasado a manos de los narcotraficantes.

Asimismo, ha intensificado las desigualdades económicas y provocado una movilidad social perversa que al quebrantar las viejas jerarquías sociales, transforma los comportamientos, en particular los de los jóvenes. Incluso puede achacársele una profundización del machismo estructural en el que incluso las mujeres se convirtieron posesiones de los narcotraficantes, buena cuenta de ello dan algunas obras literarias como la conocida "sin tetas no hay paraíso" del escritor Gustavo Bolivar (2005).

\section{Narcotráfico y cultura de la legalidad}

Una vez reseñada las fallas de Estado Nación y sus instituciones y la brecha entre Estado y sociedad, este apartado analiza con mayor detalle la relación entre los cultivos ilícitos y la cultura de la ilegalidad. Para ello se recoge el trabajo que realiza Thuomi sobre los aspectos subjetivos que permiten el desarrollo y auge del narcotráfico en Colombia. Más adelante se recoge el diagnostico de García Villegas que plantea las características de los perfiles de "incumplidores" en América Latina" en el cual encajan algunas de las características de los actores que operan en el negocio del narcotráfico y que permite analizar algunas de las dimensiones y perfiles de la relación entre los incumplidores de la legalidad y los eslabones de la cadena del narcotráfico.

El análisis de la cultura del incumplimiento de las reglas en América Latina ha sido planteado desde diferentes perspectivas. Ya se han señalado las características del marco estatal y social que fomentan la ruptura que supone en ultimas el quebranto de la legalidad.

Desde el punto de vista estratégico basado en la idea de la sociedad como un conjunto de individuos racionales y egoístas algunos economistas interesados en el estudio de las causas de la violencia, sostienen que la criminalidad se explica mejor por la falta de sanciones efectivas que por otras causas como la cultura o la pereza (García Villegas, M., 2010: 136, citando a Montenegro, 1995 y Kalmanovitz, 2001). Evidentemente un estado indolente o incapaz es fuente de impunidad, sin embargo esta no es una característica exclusiva de Colombia. Los niveles de impunidad son igualmente altos o inclusive más en otros países del entorno. Más aun el énfasis que se ha hecho en el combate del narcotráfico lo hace una de las actividades sujetas a mayores penas. Asimismo, las medidas coercitivas como la fumigación o la imposibilidad de acceder a ayudas sociales deberían actuar como desincentivos del cultivo ilícito, sin embargo, la evidencia demuestra que no funcionan así.

Desde una perspectiva más sociológica y extraída concretamente de los análisis del narcotráfico Francisco Thoumi (2015) estudia las condiciones que hicieron de Colombia un país tan susceptible a la producción y tráfico de drogas ilícitas: "Mientras que la demanda internacional o nacional es necesaria para que exista un mercado criminal, para que este se desarrolle es necesario que la sociedad tenga una estructura, normas y organismos que lo faciliten, es decir, que hagan que la sociedad sea vulnerable".

Si bien reconoce que el fenómeno del ilícito se concentrará donde es más fácil dejar de cumplir la ley, también señala que las divergencias entre lo legal y lo legítimo dependen del grado y naturaleza del capital social y de la cohesión social que exista en cada sociedad. Es decir, introduce dos conceptos que otros autores no incluyen, 
capital social y cohesión. Esto es, dos conceptos que tratan de la forma en la que se relacionan las personas entre sí y con el Estado y las características y calidad de estas relaciones. Por tanto, permite dar una perspectiva colectiva al análisis y no meramente basada en los intereses personales o egoístas.

La confianza juega un papel relevante en cuanto allí donde existe menos confianza y firmeza de los lazos sociales, la gente está menos dispuesta a cumplir. (Putnam, 1992) Ahora bien, las vulnerabilidades sociales proporcionan las razones explicativas del surgimiento de muchos males o tragedias sociales, pero las vulnerabilidades sociales no son causas, sino factores que generan riesgos que aumentan la probabilidad de que surjan los males sociales y que alimentan círculos viciosos. Las causas son una conjunción de factores desde los ambientales que permiten "el éxito" de determinados cultivos, pasando por la vulnerabilidad a la corrupción de las élites y órganos de control, hasta la existencia de una demanda internacional.

Para Thoumi, la única solución posible para Colombia empieza aceptando que concentró la producción y tráfico de cocaína porque su estructura física, normas y órganos sociales hicieron que el país fuera extraordinariamente vulnerable al surgimiento de una demanda ilegal en el extranjero". Tanto es así que otros tráficos ilícitos o emergentes a lo largo de la historia han generado procesos similares de violencia e ilegalidad, las esmeraldas o el oro, por ejemplo. Por tanto, para dar una solución al problema es necesario identificar los factores que hacen vulnerable a la sociedad, lo que implica llevar a cabo reformas sociales difíciles de implementar o de muy largo plazo.

Ahora bien, de cara a la búsqueda de soluciones y en especial de respuestas específicas que atiendan de forma diferenciada cada uno de los eslabones de la cadena de las drogas, vale la pena revisar el trabajo de García Villegas (2015). Este autor tipifica tres perfiles de incumplidores de la legalidad en América Latina: "el vivo" (listillo en España), que cree que su conducta incumplidora es una forma de mejorar sus beneficios frente a otros; el rebelde que incumple como una forma de ajustar cuentas con una sociedad injusta; y finalmente el déspota quien asume que tiene privilegios que le eximen del cumplimiento de las normas.

Esta tipología resulta útil para analizar los perfiles y justificaciones de los individuos y grupos involucrados en los eslabones del narcotráfico. Está claro que la decisión de involucrarse en una actividad ilegal está determinada por un interés personal por la consecución de recursos o poder, sin embargo, el entorno amigable a lo ilícito favorece y en algunos casos alienta la toma de decisiones personales.

Pasando al análisis de los perfiles de incumplidores, en primer lugar se encuentra, la imagen del "vivo o listillo" que sirve para tipificar al individuo que busca recursos y está dispuesto a saltarse la ley para conseguir sus fines. Puede ser la que impere tras aquellos que actúan en los eslabones de tráfico y distribución. Es la imagen popular del narcotraficante, aquella sobre la cual se basan las representaciones de las populares series de televisión.

En otro extremo encontramos el poder despótico que pasa por encima de las reglas porque se ve superior a la obligación de cumplirlas. Este tipo podría ajustarse más fácilmente a las elites corruptas que aprovechan sus privilegios para participar o facilitar el tráfico o alguno de los delitos conexos. Las sociedades altamente desiguales y con una injusta distribución de privilegios y de formas de relación con lo público son terreno abonado para elites corruptas que se aprovechan de forma patrimonial del Estado. 
Ahora bien, en el caso concreto del que se ocupa este artículo hay una de las tipologías que se adecua más claramente, sin que por ello elimine la existencia de casos de las otras dos. Se trata de la visión política del incumplimiento. Se encuentran referencias entre quienes tienen una percepción crítica del poder y del derecho en América Latina. Esta visión sigue los lineamientos de la vieja tradición iusnaturalista española que pone la justicia por encima de la ley, y del derecho a resistir al poder injusto, por encima de la obligación a cumplir como los mandatos del soberano o del funcionario. García Villegas (2010: 143) llama a este tipo "La mentalidad del incumplidor rebelde" y lo condensa en la siguiente afirmación: "Si cumplir las reglas del patrón no tiene la recompensa que es debida entonces esa recompensa hay que buscarla por medio del incumplimiento".

El cultivo de coca en Colombia está altamente ligado a una mala distribución de la tierra, concentrada en pocas manos y muchas veces obtenida a través de la coerción y la violencia. Los campesinos sin tierra, los de las regiones periféricas o de frontera, los que colonizan la selva, se han visto sistemáticamente excluidos de los mercados lícitos, de las tierras más productivas y de la representación y participación en democracia.

Se generan sistemas sociales poco cohesionadas y en las que la ausencia del Estado ha propiciado formas de organización y gestión social diferentes. Asimismo, la violencia generada por la falta de sistemas de derechos y el control ejercido por poderes fácticos y la baja productividad de las tierras selváticas cultivadas con coca a lo largo del tiempo llevan a que se produzcan migraciones, procesos continuos de colonización y desplazamiento. Por este motivo, la desconfianza entre las personas y con las instituciones es acusada. Son sociedades en continuo trasegar y que viven bajo un patrón de búsqueda continua de los medios de vida sin lograr una estabilización formal.

La coca se ha convertido, no solo en Colombia, sino también en Perú y en algunos momentos de la historia de Bolivia en un aglutinador de campesinos pobres y que se ven atacados por el Estado. Ya en los años ochenta las "marchas cocaleras" en Colombia pusieron en aprietos al gobierno nacional, que aunque no lo reconociera hasta hace poco, siempre se ha sabido incapaz de actuar en todo el territorio nacional. Ante la falta de política rural equitativa e incluyente los que tienen coca en cierta forma tienen algo que negociar para atraer la atención y los recursos del Estado o para impedir su entrada.

La guerrilla misma propició esta idea y recurrió al "clientelismo armado" (Cubides, 2006) que la convertía en una suerte de intermediario de sectores sociales cocaleros o asentados en territorios con riquezas mineras o petroleras y el Estado. En el proceso de representación y negociación la guerrilla se llevaba una parte y conseguía beneficios del Estado para poblaciones que de otra forma permanecían en el olvido. Así pues, tanto la subversión como el cultivo de coca se valían de la imagen del rebelde que actuaba fuera de la ley para atacar a los que les excluían del sistema de beneficios.

Evidentemente aquí surge el gran dilema de la lucha contra las drogas tal como ha sido planteada frente a la producción. ¿Cómo pedirle actuar en el marco de una cultura de la legalidad a quienes viven en sistemas esencialmente injustos y para los que el Estado nunca ha operado de forma positiva? 
3. El desarrollo alternativo como respuesta al problema de los cultivos ilícitos

El problema de las drogas ilícitas es en realidad la convergencia de múltiples problemáticas articuladas a través de los eslabones de una cadena que va de la siembra de cultivos ilícitos, pasando por la producción tráfico y distribución hasta llegar al consumo de las substancias psicoactivas, narcóticas y estimulantes. Dada las diferentes problemáticas relacionadas a cada eslabón de la cadena se requieren abordajes diferenciados que den soluciones sociales, sanitarias y de seguridad ajustadas a cada problemática. En el caso de la producción de cultivos ilícitos estas soluciones de orden social se encuentran en lo que se conoce como "desarrollo alternativo".

Existen diversas definiciones del desarrollo alternativo, la mayoría referidas al desarrollo alternativo como una forma de mejora sostenible de las condiciones económicas y sociales. Sin embargo, para este caso concreto la definición más apropiada es la que propone la Oficina de las Naciones Unidas Contra la Droga y el Crimen (UNODC) dado que se refiere específicamente al proceso de sustitución de cultivos ilícitos:

"Un proceso para prevenir y eliminar el cultivo ilícito de plantas que contienen substancias narcóticas y psicotrópicas a través de medidas de desarrollo rural específicamente diseñadas en el contexto de crecimiento sostenido nacional y de los esfuerzos de desarrollo sostenible en países que luchan contra las drogas, reconociendo las características socioculturales particulares de las comunidades y grupos objetivo dentro del marco de una solución completa y permanente del programa de las drogas ilícitas" (UNODC, 2015: 77b).

La definición comprende un amplio conjunto de medidas y en especial recalca la especificidad y necesidad de adaptación de las mismas a los contextos en los que se generan los cultivos ilícitos. Otro aspecto importante es la mención a la búsqueda de una solución completa y permanente del problema de las drogas. Justamente esta es la verdadera cuestión, como dar una solución permanente al problema.

Si bien, el problema de la producción de drogas no puede atenderse desde una perspectiva reduccionista, ni a través de generalizaciones, lo cierto es que en el caso de los cultivos de coca, adormidera y en algunos casos en los de cannabis su existencia es en gran medida fruto de una combinación de factores entre ellos pobreza, exclusión de los mercados, desigualdad, y en especial debilidad del Estado Nación. Este artículo se centra justamente en este último aspecto, en algunas de las múltiples facetas de la debilidad estatal, concretamente en la cultura de la "ilegalidad" ligada en este caso del cultivo de plantas ilícitas y de las estrategias con las cuales se ha tratado en el caso de Colombia.

El desarrollo alternativo fue concebido como una estrategia complementaria a las estrategias de interdicción en los países con presencia de cultivos ilícitos. El concepto fue ampliándose gradualmente y no se limitó al remplazo de los cultivos, sino que también incluyó el procesamiento y la transformación de éstos, incluyendo la comercialización a través de organizaciones productivas y alianzas con el sector privado. (UNODC OEI, 2013: 14) En suma, el objetivo es el establecimiento de un enfoque balanceado de la lucha contra las drogas en la que el reconocimiento de la existencia de condiciones sociales "facilitadoras" de la producción de drogas llevara a balancear la coerción con políticas sociales. Esto es, el reconocimiento de que a pesar de que la lucha se centra en el carácter ilegal de la actividad se asume que hay factores que inciden directamente en ella y por tanto el desarrollo Alternativo se debe 
dirigir a identificar y ayudar a concentrarse no solo en la producción concreta de narcóticos sino también en sus causas estructurales: subdesarrollo, marginalidad, pobreza entre otros.

En el caso concreto de Colombia, la política de desarrollo alternativo actual se basa en resaltar la importancia de las comunidades como espacio central de la transformación y se concentra en la generación de condiciones y oportunidades lícitas que conlleven al empleo productivo y sostenible de la mano de obra rural, que adicionalmente aporte a la superación de la pobreza de estas comunidades sustrayéndolas del circuito ilegal de los cultivos ilícitos. Esto implica idealmente el establecimiento de una base económica lícita en el ámbito regional y local, fortaleciendo el desarrollo sostenible integral, la gobernabilidad, la cultura de la legalidad, la conservación del medio ambiente y la valorización del patrimonio natural. (UNODC OEI, 2013: 14).

\section{Presencia de los cultivos de Coca en Colombia}

Tras muchos años de costosa lucha contra los cultivos ilícitos en 2016 en Colombia presentan una cifra record, 146 mil hectáreas, según el Censo anual de cultivos de coca de la Oficina de Naciones Contra la Droga y el Crimen. Un aumento del $52 \%$ frente al año 2015. Históricamente el año en el que se detectó una menor presencia de cultivos ilícitos fue el 2012 cuando bajaron a 49 mil hectáreas, es decir un tercio de la actual cifra.

A pesar del ingente esfuerzo realizado por el país a través de diversas medidas especialmente coercitivas, pero también de orden social, el objetivo de reducir las hectáreas cultivadas no ha conseguido alcanzarse. De hecho, no solo no se reducen de forma significativa las hectáreas cultivadas sino que se desplazan hacia áreas donde tienen un impacto aún mayor en términos ambientales y sociales. Tal como lo señala UNODC (2017) La afectación por cultivos de coca en áreas de manejo especial como resguardos indígenas, parques nacionales naturales 0 territorios afrodescendientes, siguen siendo una amenaza para la biodiversidad biológica y cultural del país.

En las tres categorías de áreas de manejo ambiental especial que existen en el país se registró un incremento del área sembrada con coca entre 2015 y 2016: en Resguardos Indígenas del 32\%, en Tierras de las Comunidades Negras del 45\%, y en Parques Nacionales Naturales del 27\% (UNODC, 2017: 15)

Si el impacto ambiental y geográfico de los cultivos de coca no disminuye, tampoco hay efectos notables sobre el mercado, es más, incluso cuando se reducen las hectáreas la productividad de las mismas tiende a aumentar. La producción potencial de hoja de coca fresca pasó de 454.050 toneladas métricas en 2015 a 606.130 toneladas métricas en 2016, un incremento del 33,5\% explicado principalmente por el aumento en el área productiva. Esto se debe a la aplicación de mejoras tecnológicas y de un uso más eficiente de la hoja para producir pasta base. (UNODC, 2017: 15)

Ahora bien, dado que el panorama nacional es malo en términos de la consecución de disminuciones substantivas en la presencia de cultivos ilícitos, habría que revisar el impacto de los mismos sobre la calidad de vida de los cultivadores para analizar su persistencia. Según el Reporte de Drogas de Colombia de 2016 (Observatorio de Drogas de Colombia, ODC), si se analiza el mercado global de la droga, los cultivadores solo reciben el $1,4 \%$ de los ingresos totales de la cocaína de 
todos los niveles del tráfico y en la cadena del negocio son los más vulnerables. Es más, podría esperarse que los esfuerzos de lucha contra la producción, redujeran los suministros e incrementaron los precios de la droga, pero lo que se observa es que las ganancias del negocio del narcotráfico permiten sostenerlo y crean alternativas de choque con alto grado de adaptabilidad. En suma, nos enfrentamos a un fenómeno con un alto nivel de capacidad de ajustarse a situaciones adversas en el que no hacen mella las medidas adoptadas, y que a su vez no parece basarse en su capacidad redistribuidora para generar una base de productores estable sino en su aprovechamiento de la pobreza y desigualdad crónicas como base de explotación.

Más aun, según los estudios de precios incluidos en el Monitoreo de Cultivos de Coca de 2013 en Colombia los precios de los productos derivados de la producción y transformación de coca no guardan una lógica frente a los cambios en la oferta y la demanda. Por tanto cobra fuerza hipótesis sobre la prevalencia de una estructura de mercado de tipo monopsonio lo cual lleva a un control del precio con niveles más bajos en relación a aquellos que se pactarían en un mercado competitivo.

De hecho, se estima que en 2015 alrededor de 74.500 hogares con un promedio de 5 miembros, percibieron ingresos por actividades de producción. Cada integrante de hogar podría recibir alrededor de 1.180 dólares al año frente a los 5805 de renta per cápita del país (SIMCI, 2017). Valga apuntar que en uno de los países más desiguales del mundo, esta cantidad es superior a la renta media de un hogar campesino minifundista dedicado a los cultivos lícitos.

4.1. La política dicotómica de la contención de los cultivos ilícitos: entre la seguridad y el desarrollo social

En términos generales la estrategia de erradicación o de lucha contra los cultivos ilícitos se basa en dos tipos de estrategias: las de interdicción y las de desarrollo alternativo. Las primeras estrategias corresponden a aquellas que buscan la destrucción de los cultivos a través de la fumigación, o la erradicación manual.

Las medidas de desarrollo alternativo se concentran, en términos generales, en la búsqueda de la sustitución de cultivos. Dicha sustitución se promueve a través de la generación de proyectos productivos individuales y/o colectivos y de transferencias condicionadas. Adicionalmente existen otras medidas de intervención en los territorios a través de inversiones en infraestructuras y dotaciones.

\subsection{Programas de Desarrollo Alternativo}

En Colombia la Ley 30 de 1986, conocida como Estatuto Nacional de Estupefacientes (ENE) determina que todos los eslabones de la cadena de las drogas ilícitas son ilegales y constitutivos de delito. Concretamente la Ley señala:

"El que sin permiso de autoridad competente cultive, conserve o financie plantaciones de marihuana o cualquier otra planta de las que pueda producirse cocaína, morfina, heroína o cualquier otra droga que produzca dependencia, o más de un (1) kilogramo de semillas de dichas plantas, incurrirá en prisión de cuatro (4) a doce (12) años y multa de diez (10) a cuatrocientos (400) salarios mínimos mensuales. Si la cantidad de plantas de que trata este artículo excediere de veinte (20) sin sobrepasar la cantidad de cien (100), la pena será de uno (1) a tres (3) años de prisión y multa en cuantía de uno (1) a cuarenta (40) salarios mínimos mensuales." 
Es decir que el cultivo es ilegal y supone penas muy significativas de cárcel, sin embargo, poco después de la aprobación del Estatuto se iniciaron los primeros programas de sustitución de cultivos ilícitos. En 1987 se hicieron las primeras intervenciones en los departamentos de Cauca y Nariño, en el sur del país, a través de un enfoque multisectorial coordinado bajo la estrategia de Naciones Unidas. En 1990 este programa se amplió a los departamentos de Guaviare, Caquetá y Putumayo (Programa de las Naciones Unidas para la Fiscalización Internacional de las drogas). En 1993 el gobierno impulsó el Plan Nacional de Rehabilitación a través de programas para la sustitución de amapola en los departamentos de Tolima y Cauca.

La estrategia se reformuló en 1995 a través del Programa Nacional de Desarrollo Alternativo (PLANTE) una intervención territorial focalizada. Este programa fue pionero en usar la presencia de cultivos ilícitos como criterio de focalización municipal y se financió a través de un préstamo del Banco Interamericano de Desarrollo. La estrategia de erradicación usada fue la de erradicación paulatina, -"a través de acuerdos con los pequeños productores, estrategia que llevó a lo que denominaron -desarrollo con coca-, donde se sustituían cultivos y a la vez se cultivaba coca"- (DNP, 2012: 10)

Este Plan sufrió grandes problemas de gestión que lo hicieron prácticamente inviable en su primera fase. La segunda fase, se caracterizó por una pésima toma de decisiones debida al desconocimiento de las características sociales del problema de los cultivos ilícitos (Uribe, S., 2002). Ninguna de las dos fases consiguió resultados notables.

A la finalización del PLANTE se instituyó entre 2001 y 2003 y de forma paralela al Plan Colombia el Programa Campo en Acción, que estableció los primeros pactos de erradicación voluntaria con cultivadores, pero que por fallos en la gestión se vio limitado en su alcance.

El Plan Colombia, por su parte (1998-2015), determinó un giro de 180 grados que no solo consolidaba las directivas de la política antidrogas norteamericana sino que unía en una misma estrategia la lucha antinarcóticos con la lucha antiterrorista y antisubversiva. El Plan Colombia tenía un componente militar, el mayoritario, que redefinió el equilibrio bélico del conflicto armado en Colombia. El componente social por su parte, era minoritario y estaba subordinado a la estrategia militar y sobre todo a su lógica securitaria. (Rodríguez, E., 2015). El Plan se desarrolló en tres etapas las dos primeras de ellas durante los gobiernos de Andrés Pastrana y Álvaro Uribe. Entre 2000 y 2007 la mayor parte de las ayudas del componente social se destinó a Desarrollo Alternativo (DNP, 2015). Fue en este periodo donde la guerra alcanzó su mayor impacto humanitario a través de masacres, combates y ataques indiscriminados y el desplazamiento de millones de colombianos. El sector rural periférico colombiano quedó en medio del fuego cruzado y bajo la dominación de los grupos en conflicto. Tanto guerrillas como paramilitares se beneficiaban de la coca y propiciaron su cultivo, aunque sus finanzas se diversificaron con otros negocios extractivos como la minería del oro, y la ganadería.

Junto con los programas de desarrollo alternativo en el Plan Colombia la estrategia de fumigación con "glifosfato" se reforzó, lo cual generalizó la estrategia de garrote o zanahoria. Esto es, la dicotomía entre la adopción de los planes y propuestas gubernamentales o la destrucción de los cultivos y criminalización del cultivador como infractor de ley y aliado de grupos "narcoterroristas". 
De cualquier forma, a pesar, de la capacidad coercitiva del Plan Colombia, y tal como reflejan las cifras las hectáreas cultivadas no disminuyeron, en 2007 sobrepasaban las 90 mil hectáreas e incluso habían aumentado frente a 2003.

En 2006 se creó el Plan Nacional de Consolidación territorial, una estrategia que buscaba llevar el Estado a los territorios en los cuales no había estado presente o solo lo había hecho a través de la estrategia militar, concretamente en el caso de la substitución de cultivos ilícitos su objetivo estaba "concentrado en la generación de condiciones y oportunidades lícitas para el empleo productivo y sostenible de la mano de obra rural" (CONPES).

Este Plan creó varias estrategias que han permanecido en el tiempo aunque con importantes variaciones especialmente en su financiación para atender la problemática y el impacto de los cultivos ilícitos; La primera de ellas la erradicación forzada a través de erradicación manual.

Dado que el uso del glifosato había abierto un gran debate y más aún una controversia jurídica internacional con Ecuador por los supuestos efectos nocivos de este herbicida. La erradicación forzosa se implementó a través de "batallones" de erradicadores manuales, oficialmente denominado como Grupo Móvil de Erradicación, que destruían una a una las plantas. En el marco del conflicto armado esta estrategia abrió un nuevo frente de combate e incentivó el uso de minas antipersonales por parte de los grupos armados no estatales.

En entrevistas realizadas por la autora en terreno en el marco de una evaluación para un organismo multilateral (UNODC; 2013) los campesinos acogidos ahora al programa de desarrollo alternativo relataban como los grupos guerrilleros les daban cursos para instalar las minas para proteger sus cultivos; y lo que es más grave, otros de sus vecinos relataban que ante la falta de empleo se habían convertido en erradicadores. Literalmente se trataba de un campo de guerra que convirtió a iguales en la pobreza en enemigos sin que realmente existiera una diferencia ideológica ni de objetivos últimos entre unos y otros. Más aun, algunos de los erradicadores habían vuelto luego al cultivo de coca y después se habían acogido a programas de erradicación manual coincidiendo en estos con los que siempre mantuvieron sus cultivos.

Paralelo al proceso de erradicación manual, se creó el programa de Familias guardabosques que consistía en "transferir condicionalmente un apoyo económico directo a familias campesinas, indígenas y afrodescendientes para la recuperación y conservación de ecosistemas, el uso sostenible de los recursos naturales, la implementación de bienes y servicios ambientales y la generación de ingresos alternativos en zonas social y ambientalmente estratégicas" (DNP, 2003). La particularidad de este programa fue que no se focalizó en los cultivadores de coca sino especialmente en aquellas familias que se asentaban en territorios donde había "riesgo" de coca. Si bien este criterio desde un punto de vista de prevención y atención integral tiene sentido, por el otro también quedó abierto a la indefinición del concepto "riesgo".

Dado que la presentación de resultados positivos es imperiosa para cualquier gobierno, el criterio del "riesgo" se convierte en una ocasión para aplicar las políticas en zonas donde no existen las condiciones que propician el narcotráfico y si las que permiten la pervivencia de la economía lícita y por tanto donde de los incentivos tienen mayores resultados. Un buen ejemplo es la implementación del programa en zonas donde históricamente no ha habido cultivos como por ejemplo en las islas en el Caribe. No se señala que el objetivo fuera incorrecto, pero sí que no debería 
corresponderse a la estrategia antinarcóticos sino a la estrategia de desarrollo rural del país. Del total de las familias cobijadas por el programa el $69.8 \%$ nunca habían tenido contacto con cultivos ilícitos y el 30.2\% si lo habían tenido. (UNODC, 2011:10)

Una tercera estrategia fue la de "proyectos productivos" (previa erradicación voluntaria) y la ya señalada erradicación forzosa para quienes no erradicaran voluntariamente. Los proyectos productivos buscaban generar oportunidades para otros procesos productivos entre campesinos que se comprometieran a no volver a sembrar ilícitos. En este caso destaca la potenciación de la creación de cooperativas y el enfoque territorial y no individual de los proyectos. El programa entendía de forma acertada que es necesaria la transformación comunitaria y por tanto buscaba crear condiciones para favorecer dicho proceso.

Tanto en la estrategia de Familias Guardabosques como en la de Proyectos Productivos se visibilizan esfuerzos en su diseño por trabajar desde una óptica de fomento de la cultura de la legalidad aunque una vez revisados los instrumentos puestos en marcha queda en evidencia que no existen herramientas específicas para ese objetivo.

Actualmente, el Programa Integral Nacional de Sustitución de Cultivos de uso Ilícito (PNIS) es parte de la implementación de los Acuerdos de la Habana entre el Gobierno de Colombia y la guerrilla de las FARC. El Acuerdo de paz señala que la superación del problema del narcotráfico no debe limitarse a la erradicación de cultivos, sino al cambio en las condiciones de vulnerabilidad en los territorios, su transformación. Esto supone la articulación de estrategias en el territorio para conseguir su transformación integral. Esta apuesta se corresponde con el exhaustivo diagnóstico de las causas y consecuencias de la confrontación armada que se realizó a lo largo del proceso de diálogos y que dio lugar a los siete puntos del Acuerdo.

En el diagnóstico de la estructura y causas del conflicto armado, más que atender a la cultura de la ilegalidad, se discutieron los fallos del Estado, su histórica ausencia de los territorios periféricos o su presencia fragmentada y con ello la debilidad del Estado de Derecho con el que no tienen relación alguna buena parte de los colombianos. La presencia de los cultivos ilícitos es una más de las consecuencias del fallo del Estado ligado a la presencia de incentivos para el crimen organizado que se analizarán más adelante.

La sustitución de cultivos que se propone a día de hoy pasa por la transformación de los territorios, y por tanto requiere articularse con el concurso de las comunidades. Tal como lo señala la Fundación Ideas Paz (FIP 2017) el PNIS es la cara más visible de la implementación del Acuerdo de Paz con las FARC en muchas veredas y municipios con coca donde la intervención del Estado resultó imposible en el pasado por la confrontación armada y el control de la guerrilla.

En estos territorios, con el acompañamiento de las FARC, el Gobierno ha venido convocado a las comunidades y la institucionalidad local para construir acuerdos colectivos que permitan avanzar en la sustitución. La firma de acuerdos con las comunidades permite iniciar procesos que a través de subsidios, apoyo técnico y social faciliten a las familias el paso a cultivos lícitos. Una estrategia que sigue el modelo de los proyectos productivos pero incorporando componentes de dialogo y compromiso con las comunidades para incorporarlas a la voluntad de transformar el territorio y que además bebe de las lecciones que dejaron los anteriores programas que se apoyaban en sistemas de transferencias condicionadas. 
Este proceso se ha iniciado en el marco de una fuerte presión internacional, dado que los recientes cambios en el gobierno de EEUU han llevado a urgir, nuevamente, al país a disminuir los cultivos a través de las medidas coercitivas como la fumigación. Evidentemente el uso de la misma daría al traste con cualquier intento de acuerdo con las comunidades y con la posibilidad de integrarlas al esfuerzo por consolidar el Estado. De esta manera, según la Fundación Ideas Paz (FIP 2017) la demanda de soluciones inmediatas se contrapone con la necesidad de apostarle a un esfuerzo sostenido enfocado en mejorar la calidad de vida de los campesinos y el desarrollo rural, con el riesgo de que las respuestas inmediatas terminen consumiendo los recursos necesarios para implementar las medidas de largo plazo. Está claro que esta tarea no será fácil, ni rápida.

\section{Cultura de la legalidad y substitución de cultivos ilícitos: Evidencia Empírica}

Hasta ahora se han descrito los programas con los que históricamente ha intentado realizarse la sustitución de los cultivos ilícitos en Colombia. A continuación se realiza un análisis de la relación entre la debilidad del Estado y sus fallos y la cultura de la ilegalidad.

Del apartado anterior se recogen dos conclusiones: la primera es la incapacidad de los programas que han existido en conseguir transformaciones permanentes, así, como su incapacidad para generar un arraigo a los mismos entre la población. Una de las claves para entender esta incapacidad está en lo que ya en 2002, un consultor en desarrollo alternativo señalaba: "Durante los últimos 12 años los gobiernos han impuesto soluciones sin entender que hay que concertar las mismas con el campesinado..." más aun, afirmaba "se debe reflexionar sobre el daño que se hace el Estado a si mismo cuando no cumple con sus promesas, pierde su credibilidad y por ende su legitimidad" (Uribe, S., 2002).

El segundo de los problemas emana de la necesidad y presión por obtener resultados por encima de atender la complejidad de la problemática. La sustitución enfrenta múltiples desafíos vinculados a la débil articulación del Estado en el territorio, la relación del centro con la periferia, la distribución desigual de los recursos, la permanencia de actores armados ilegales y la generación de expectativas en un contexto de austeridad y competencia entre diversos actores (FIP, 2017: 16)

En este apartado se analiza la evidencia existente en materia de fortalecimiento de la cultura de la legalidad a través de los diferentes programas que han tenido lugar en el país con el objeto de acabar con los cultivos ilícitos.

A pesar de la evidente importancia de la creación de una cultura de la legalidad, o del acercamiento o impulso de la misma entre las personas de cara al esfuerzo de erradicación de una actividad ilícita, este componente no siempre ha estado presente y visible en los programas. De hecho, es en los últimos años en los que en el marco del trabajo holístico con los "territorios" ha encontrado algún encaje. Asimismo, es importante señalar que no hay una gran oferta de trabajos académicos que analicen o levanten evidencia sobre este fenómeno. La popularidad de los diagnósticos sobre la existencia del narcotráfico no encuentra contraparte en el estudio de las repercusiones sociales, o psicosociales o comportamentales de los colectivos objetivo de la política de desarrollo alternativo.

Un trabajo reciente pero que recoge una evaluación muy completa del impacto de los programas para llevar el Estado a las regiones es el de Claudia López (2016). La autora analiza de forma sistemática las políticas de los últimos 30 años y encuentra que, por ejemplo, donde coinciden la herencia del ya reseñado Plan Nacional de 
Rehabilitación y la intervención antinarcóticos del Plante se sustituyen unos efectos negativos por otros en la dimensión de coerción, se intensifican los efectos adversos en la dimensión de erradicación y se mantienen los de baja participación en toda las elecciones en la dimensión de legitimidad". Es decir que ni se consiguieron resultados en términos de erradicación ni tampoco se consiguió alentar la participación política, sintomática de una relación viva con el Estado.

También evidencia que la coincidencia de Programas de Desarrollo Productivo con el Plan Colombia disminuye la violencia pero genera más resistencia a la autonomía fiscal y la participación electoral.

En general, el trabajo concluye que el balance de los programas presidenciales para llevar el Estado a las regiones de los últimos treinta años ha sido limitado, cuando no contraproducente. Establece que si bien los programas sin duda han fortalecido la estatalidad colombiana general, sin embargo, los municipios intervenidos por los programas no han seguido la misma tendencia "ciertas formas de violencia se crecen u otras se reciclan, el recaudo de impuestos y capacidad de inversión local usualmente se deterioran, así como la calidad de la gestión local y la participación electoral en los municipios intervenidos" (López, C., 2016: 311)

Este trabajo resulta interesante en cuanto a falta de evaluaciones de impacto de los programas determina dimensiones de análisis que pueden ser útiles en ausencia de otros datos.

Otra fuente de información de evidencia empírica son las evaluaciones independientes que se han desarrollado sobre los Programas de Desarrollo Alternativo y particularmente sobre los programas de proyectos productivos y familias guardabosques.

Dichas evaluaciones dejan evidencias que aunque limitadas, dadas las restricciones de muestreo y la aplicación de metodologías esencialmente cualitativas dan una buena idea sobre la calidad e impacto de estos programas.

En la evaluación realizada por UNODC sobre los programas de desarrollo alternativo de un periodo de 10 años destacan algunos hallazgos como el desgaste de la participación social dada la falta de continuidad de los programas o el limitado éxito de los proyectos productivos implantados. La mayoría de ellos incapaces de remplazar los ingresos y facilidades comerciales de la coca de forma permanente.

A pesar de ello los beneficiarios de los proyectos reconocen y valoran el apoyo que el Programa de Desarrollo Alternativo (PDA) y UNODC les ha brindado y tienen un alto nivel de confianza en esta institución, pero también cierto grado de dependencia de los técnicos de Naciones Unidas. A pesar de tratarse de comunidades rurales el monocultivo de coca ha debilitado los conocimientos agrícolas para la implementación de otros cultivos y eso hace que sea necesaria la presencia de os apoyos técnicos por periodos más largos que los establecidos en los programas. La discrepancia entre la lógica de proyecto y su calendarización y la necesidad de apoyo social y productivo hacen que los lazos de confianza creados durante un programa se debiliten y extingan ante el súbito "abandono" a la finalización de los programas.

El modelo de consolidación territorial es de carácter concéntrico se comienza la intervención desde el centro poblado y se va consolidando dentro del territorio. Este proceso puede tomar varios años y por lo tanto si se deja de atender a las familias y no existe una estrategia expedita para que otros programas lo apoyen las familias que queden desatendidas estarán nuevamente en riesgo de regresar a lo ilícito, a ser parte 
de grupos armados o bandas emergentes o regresar a la condición de pobreza en la que se encontraban antes de la intervención (UNODC, 2014: 63)

Otra evidencia muy relevante es que en la mayoría de los casos no se ha percibido un pleno reconocimiento por parte de las comunidades de la fuente de financiación del PDA por parte del gobierno colombiano; a pesar de procesos de socialización y sensibilización realizados, es todavía escasa la notoriedad de la Unidad de Consolidación Territorial del gobierno alcanzada entre los beneficiarios como entidad encargada del PDA (UNODC, 2014: 62) Los campesinos atendidos por los programas no reconocen que estos son un esfuerzo del gobierno por brindar alternativas. Al ser ejecutado por otros actores bien sea Naciones Unidas u ONG solo establecen relaciones de confianza con estos y no con la institucionalidad estatal que es la que debe entrar y asentarse en el territorio. El Estado a pesar de su esfuerzo sigue siendo un extraño y una fuente de desconfianza.

Específicamente en materia del establecimiento de lazos de confianza, una de las evaluaciones concluye que los PDA deberían incluir la atención del tema psicosocial y que este no ha sido incluido nunca. De hecho, señalan que en comunidades afectadas por el conflicto es indispensable generar confianza entre las personas, para adelantar con éxito los proyectos. Es fundamental lograr un acompañamiento más prolongado y diferencial y un fortalecimiento de los sistemas de organización (UNODC, 2014: 87)

Finalmente se reseña un trabajo que quizás es el que más concretamente ahonda en la cuestión de la cultura de la legalidad. Ibáñez y Martinsson (2013) realizaron diferentes estrategias para obtener evidencia empírica sobre cambios actitudinales en las personas que habían participado en PDA.

A pesar de los escasos resultados objetivos, su experimento permite demostrar cambios positivos en actitudes personales: mayor tendencia a actuar de forma honesta y demuestran avances sociales cuando se interviene con enfoques positivos y de recompensa.

El trabajo consistió en aplicar en el terreno dinámicas de juegos en las que una conducta de trampa permitía ganar fácilmente. De forma muy resumida los resultados mostraban que aquellas personas que habían accedido a intervenciones de tipo PDA recurrían a la trampa con menos frecuencia que los que no lo habían hecho y que la existencia de incentivos para actuar de forma legal determinaba cambios en la conducta.

Si bien el resultado es relevante y apoya la conclusión, esta es limitada y de difícil generalización, por lo cual persiste de la necesidad de potenciar la inclusión de estrategias para fortalecer los vínculos de confianza y las conductas legales

\section{Conclusiones y perspectivas}

El déficit en la construcción de un sistema social cohesionado y de una institucionalidad incluyente es uno de los factores determinante en la producción de cultivos ilícitos a gran escala en Colombia.

Tras revisar los instrumentos, puesta en marcha y resultados de los programas de desarrollo alternativo en el país se ha puesto de manifiesto que en general que estos no han sido consistentes en el diagnóstico y abordaje de las fallas en la cultura de la legalidad, especialmente al determinarse desde un enfoque que se basa en la seguridad del Estado como objetivo último y de la implementación centrada en la 
búsqueda de productos sustitos de la coca como instrumento. La coca se ha afianzado en el país porque este cuenta con las condiciones facilitadoras para la ilegalidad, por tanto su combate pasa por atender no al producto como tal sino al conjunto de los problemas que determinan su existencia.

A la falta de resultados y de enfoques específicos que alimenten la confianza entre personas y con las instituciones, la cohesión y la cultura de la legalidad se suma el efecto perverso de la falta de resultados de los programas de Desarrollo Alternativo que profundizan la desconfianza en las instituciones. La falta de resultados, de

A pesar de los escasos estudios académicos sobre la cultura de la legalidad en el marco del desarrollo alternativo, se han encontrado evidencias a favor de enfoques basados en la generación de estímulos positivos para el ingreso a legalidad pero requieren acompañarse de medidas efectivas para mejorar la calidad de vida de las familias campesinas y en especial para conseguir que estas mejoras sean sostenibles en el largo plazo. Asimismo es necesario realizar evaluaciones para garantizar el cumplimiento de objetivos en este sentido a mediano y largo plazo. Desde luego tendría sentido que se desarrollara una propuesta basada en la teoría del cambio para fundamentar los programas de desarrollo alternativo.

En el marco del entorno cambiante hay razones para creer que es posible mejorar la implementación de enfoques amplios en los que se incluya de forma consistente la cultura de la legalidad como herramienta y objetivo.

Ahora bien, el contexto para el futuro de los procesos de desarrollo alternativo en el marco de la implementación de los Acuerdos de Paz de la Habana es incierto tanto a nivel internacional como nacional.

En los últimos años se ha abierto un debate global sobre el cambio de la política de drogas con resultados escasos aun. En 2016 tuvo lugar la Sesión Especial de Naciones Unidas sobre Drogas, aunque dio escasos resultados en materia de cambio del marco normativo internacional sí que abrió la puerta a la flexibilización de la interpretación del mismo. Esto es una oportunidad para que Colombia tenga mayor autonomía en la determinación de su política antidroga y en el abordaje social de la misma. Sin embargo la elección de Donald Trump ha traído de nuevo presión por obtener resultados en disminución de hectáreas con medidas de coerción que imposibilitan cualquier instrumento que intente un cambio en la relación con la legalidad.

Finalmente y aunque no ha sido objeto de este artículo no se puede dejar de señalar, otro de los factores que inciden en la articulación de estrategias sostenibles a largo plazo y con una mayor capacidad de incidencia en aspectos sociales: la crisis de ingresos que está sufriendo el país. La caída en los precios del petróleo y en general las materias primas han limitado la capacidad del estado para cubrir las políticas de implementación de los acuerdos de paz. Lo que se une a la inestabilidad política del país ante las próximas elecciones presidenciales en las que se define el futuro del postconflicto. 
Bibliografía

BOLIVAR, G. (2005), Sin tetas no hay paraíso, DeBolsillo, Bogotá.

COLOMBIA. DEPARTAMENTO NACIONAL DE PLANEACIÓN (2011), Bases del Plan Nacional de Desarrollo 2010 - 2014.

DEPARTAMENTO NACIONAL DE PLANEACIÓN (2003), CONPES 3218 Programa de Desarrollo Alternativo 2003-2006.

DEPARTAMENTO NACIONAL DE PLANEACIÓN (2010), Documento Conpes 3669 Política nacional de erradicación manual de cultivos ilícitos y desarrollo alternativo para la consolidación territorial. Bogotá.

DEPARTAMENTO NACIONAL DE PLANEACIÓN (2015), Evaluación del Programa de Familias Guardabosques y Grupo Móvil de Erradicación; SINERGIA.

DEPARTAMENTO NACIONAL DE PLANEACIÓN (2015), Plan Colombia: Balance de los 15 años. Disponible en:

https://colaboracion.dnp.gov.co/CDT/Sinergia/Documentos/PLAN COLOMBIA Bolet in 180216.pdf

DEPARTAMENTO NACIONAL DE PLANEACIÓN; UNIÓN TEMPORAL ECONOMETRÍA CONSULTORES (2012), Evaluación del Programa de Familias Guardabosques y Grupo Móvil de Erradicación. Disponible en:

http://www.prosperidadsocial.gov.co/inf/doc/Evaluaciones $\% 20$ de $\% 20$ Programas $\% 20$ $\%$ 20Histrico/2012-

EVALUACION\%20PROGRAMA\%20FAMILIAS\%20GUARDABOSUQES\%20Y\%20G RUPO\%20MOVIL\%20DE\%20ERRADICACION.pdf

THOUMI, F. (2012), ¿El "problema" del control de drogas es institucional o de política?. Disponible en:

http://www.mamacoca.org/FSMT sept 2003/es/doc/thoumi francisco control asunt o institucional o pol es.htm

FUNDACIÓN IDEAS PAZ (FIP) (2017), ¿En qué va la sustitución de cultivos ilícitos? Principales avances, desafíos y propuestas para hacerles frente, Primer Trimestre

GARCÍA PINZÓN, B. (2015), Cooperación y seguridad en la Guerra contra las drogas, Universidad Nacional de Colombia, Bogotá.

GIZ (2010), Rethinking the Approach of Alternative Development, Principles and Standards of Rural Development in Drug Producing Areas

IBAÑEZ, A. y ARIAS, M. (2014), "Conflicto armado en Colombia y producción agrícola. ¿Aprenden los pequeños productores a vivir en medio del conflicto?” En M. Arias et al. Costos económicos y sociales del conflicto en Colombia. ¿Cómo construir un postconflicto sostenible?, Uniandes, Bogotá. 
IBAÑEZ, M. y MARTINSSON, P. (2013), "Curbing coca cultivation in Colombia - A framed field experiment", Journal of Public Economics; Volume 105, September 2013, Pages 1-10.

LOPEZ, C. (2016), Adiós a las FARC ¿y ahora qué? Construir ciudadanía, Estado y mercado para unir las tres colombias, Editorial Debate, Bogotá.

MATHIEU, H. y NIÑO, C. (edits) (2013), From Repression to regulation: Proposals for drug policy reform, Friederich Ebert Stiftung, Bogota.

NACIONES UNIDAS (2015), Action Plan on International Cooperation on the Eradication of Illicit Drug Crops and on Alternative Development (General Assembly resolution S-20/4 E).

OBSERVATORIO DE DROGAS DE COLOMBIA (2017), Reporte de Drogas de Colombia 2016, Ministerio de Justicia, Bogotá.

PECAUT, D. (2006), Crónica de cuatro décadas de política colombiana, Norma, Bogotá.

PECAUT, D. (1996), "Presente, Pasado y Futuro de la Violencia en Colombia.", Desarrollo Económico, Vol. 36 N.144, pp. 891-930.

PECAUT, D. (2004), "Hacia la Desterritorialización de la Guerra y de la Resistencia a la Guerra"; en VARIOS (2004) Dimensiones Territoriales de la Guerra y la Paz, Universidad Nacional de Colombia, Bogotá.

RODRÍGUEZ, E. (2015), Narcotráfico y Conflicto armado en Colombia; en IBAÑEZ, $\mathrm{J}$, y otros, Criminalidad Organizada en conflictos internos, Technos/Universidad, Barcelona.

TICKNER, A. (2001), "La guerra Contra las Drogas: Las Relaciones ColombiaEstados Unidos Durante la Administración Pastrana”, en ESTRADA, J. (ed.), Plan Colombia: Ensayos críticos, Universidad Nacional de Colombia, Bogotá.

THOUMI, F. (2015), Debates y paradigmas de las políticas de drogas en el mundo y los desafíos para Colombia - Editorial Academia Colombiana de Ciencias Económicas.

UNODC (2011), Encuentro Nacional Programa Contra Cultivos Ilícitos, UNODC, Bogotá.

UNODC (2015), Principios guías sobre desarrollo alternativo de Naciones Unidas.

UNODC (2015), World Drug Report

UNODC, Oficina de Evaluación independiente (2013), Informe de Evaluación Independiente Programa de Desarrollo Alternativo Colombia, Bogotá.

URIBE, S. (2002), “Desarrollo Alternativo”, Revista Semana, 16/06/2002. 
WOLA (2006), "In dubious battle: fumigation and the U.S. War on Drugs in Colombia". Memo to the Congress. Disponible en:

http://www.wola.org/publications/in dubious battle fumigation and the us war on drugs in colombia 